\title{
Neodymium yttrium aluminium garnet laser photocoagulation for major haemorrhage from peptic ulcers and single vessels: a single blind controlled study
}

\author{
I A MACLEOD, P R MILLS, J F MACKENZIE， S N JOFFE， R I RUSSELL， D C CARTER
}

\begin{abstract}
A prospective single blind controlled trial was performed to assess the efficacy of the neodymium yttrium aluminium garnet laser in the management of patients bleeding from peptic ulcers and single vessels. Over 20 months 184 patients were found at endoscopy to be bleeding from peptic ulcers and single vessels; 130 of these failed to meet the criteria for entry into the study, but their bleeding stopped with conservative management. Forty-five patients entered the study: in all 25 who were bleeding from ulcers with spots the bleeding stopped irrespective of the treatment allocated. Twenty patients were bleeding from arteries. Eight were allocated to placebo treatment, and all later underwent emergency surgery for further haemorrhage. Twelve were allocated to receive laser treatment, and only one of eight who actually received it required surgery $(p<0.002)$.

It is concluded that laser treatment reduces the incidence of further haemorrhage and the need for emergency surgery, but the technique is difficult and not always applicable.
\end{abstract}

\section{Introduction}

The accepted current method of arresting continuous or further haemorrhage from peptic ulceration is emergency surgery. Many patients, however, are elderly with coexistent cardiorespiratory disease, and there is appreciable mortality from the operation. ${ }^{1-5}$ Dronfield $^{5}$ analysed the causes of death in a series of patients bleeding from the upper gastrointestinal tract and found that the commonest causes of potentially avoidable death were postoperative complications. If continuous or further haemorrhage could be prevented or treated without recourse to surgery then mortality might be reduced. Photocoagulation with a neodymium yttrium aluminium garnet laser is a potential non-operative method of arresting bleeding, ${ }^{6}$ and we present the results of a single blind controlled study to assess its efficacy in the management of patients with major haemorrhage from peptic ulcers and single gastroduodenal vessels.

\section{Patients and methods}

PATIENTS

Patients admitted to Glasgow Royal Infirmary from 1 October 1980 to 31 May 1982 because of acute non-variceal upper gastrointestinal

\footnotetext{
University Department of Surgery and Gastroenterology Unit, Royal Infirmary, Glasgow G4 0SF

I A MACLEOD, FRCS, research fellow

P R MILLS, MRCP, senior registrar in medicine and gastroenterology

J F MACKENZIE, MRCP, consultant physician

$S$ N JOFFE, MD, FRCS, professor of surgery

R I RUSSELL, MD, FRCP, consultant gastroenterologist

D C CARTER, MD, FRCS, professor of surgery
}

haemorrhage were considered for the study. Patients admitted for other reasons who developed upper gastrointestinal bleeding in hospital were not included.

CRITERIA FOR ENTRY INTO THE TRIAL

Patients were eligible for the study if gastrointestinal bleeding had occurred in the preceding 24 hours and they had active arterial haemorrhage at endoscopy or at least one of the following clinical criteria was fulfilled. (1) Presence of shock on or after admission as a result of blood loss. Shock was defined as the presence of pallor, cold and clammy hands and feet, and a systolic blood pressure of less than $100 \mathrm{~mm} \mathrm{Hg}$ with a pulse rate greater than 100 beats/minute. (2) An initial or subsequent haemoglobin concentration of less than $10 \mathrm{~g} / \mathrm{dl}$. (3) Blood transfusion judged necessary in the preceding 24 hours by the clinician responsible for management.

Patients fulfilling the clinical entry criteria had also to satisfy one of the following endoscopic criteria before entry to the trial. (1) Presence of a gastric or duodenal ulcer considered to be the source of haemorrhage with either an artery or a red, blue, or black spot in the ulcer base. (2) Presence of a single vessel (bleeding or not) that was considered to be the source of haemorrhage.

All endoscopies were carried out by experienced staff serving on the emergency endoscopy rota. The trial protocol specified that fresh clot could be washed away to define the underlying lesion, but no attempt was made to dislodge firm clot. Re-endoscopy could be performed in patients with firm clot if bleeding recurred.

The protocol was approved by the district ethical committee, and all patients gave written informed consent before inclusion.

\section{TRIAL DESIGN}

On fulfilling the entry criteria the patient was allocated by random card selection to receive laser or placebo treatment. Patients with gastric ulcers, duodenal ulcers, and single vessels were regarded as separate groups for randomisation purposes. To ensure uniformity of treatment laser photocoagulation was carried out by one clinician (I MacL), who did not divulge whether it had been used and did not play any part in subsequent clinical management. After endoscopy had been done the patients were returned to the care of the referring clinician with information defining the source of bleeding and notification that they had entered the trial. All patients received cimetidine $(200 \mathrm{mg}$ as an intravenous bolus injection six hourly for 24 hours, then $1 \mathrm{~g}$ daily by mouth for one month). All decisions on further management were the responsibility of the clinician in charge of the patient. If an episode of further haemorrhage was suspected and fresh bleeding was not overt endoscopy was repeated to determine whether bleeding had occurred and to define its source.

METHOD OF ASSESSMENT

Success or failure of laser or placebo treatment was assessed by one of two independent observers, who were unaware of the treatment given and were not concerned in the clinical management. The following criteria were used to assess outcome. (1) Further haemorrhage after the index bleeding episode as indicated by at least one of the following: overt haematemesis; passage of fresh blood per rectum; aspiration of over $100 \mathrm{ml}$ fresh blood from the nasogastric tube; fall in haemoglobin concentration of over $3 \mathrm{~g} / \mathrm{dl}$ within 48 hours; shock in the presence of continuing melaena; the presence of fresh blood in the stomach or duodenum at repeat endoscopy when further bleeding was suspected clinically. (2) Emergency surgery to arrest continuing or further haemorrhage. (3) Death of the patient. 
EXCLUSIONS AND WITHDRAWALS FROM THE TRIAL

Patients were excluded from the study if they were unwilling to give informed consent or if at endoscopy the pylorus was too narrow to permit duodenal intubation when bleeding was judged to be originating from duodenal ulceration. Patients were withdrawn from the study if the rate of blood loss was such that the delay entailed by endoscopy was judged to constitute an unacceptable risk to life.

\section{ENDOSCOPY AND LASER TECHNIQUE}

At endoscopy intravenous diazepam was used for sedation and hyoscine butylbromide (Buscopan) $20-40 \mathrm{mg}$ for gastric relaxation. If gastric lavage was necessary this was performed with a 36 French gauge gastric tube and 2-5 1 cold water. Diagnostic endoscopy was performed with Olympus fibrescopes (GIF-D3, K, or T; Key Med).

The endoscope used for photocoagulation was a twin channel Olympus fibrescope (TGF-2D; Key Med) with a Schott glass filter inserted into the eye piece to protect the operator's eye from back scattered and reflected radiation. The laser was a prototype neodymium yttrium aluminium garnet (wavelength $1060 \mathrm{~nm}$ ) laser (Barr and Stroud, Glasgow) with a helium neon laser incorporated (wavelength $628 \mathrm{~nm}$ ) to provide a marker beam. The laser beam was launched into a $400 \mu \mathrm{m}$ flexible glass fibre (Pilkington PE, Lytham St Annes) encased in a $2.5 \mathrm{~mm}$ polyethylene cannula, which also served as a conduit for coaxial carbon dioxide. The laser was operated in a continuous wave mode, and the fibre exit beam had a divergence of $10^{\circ}$. For photocoagulation peak powers of $70-90 \mathrm{~W}$ were used with total incidental energies of roughly $50 \mathrm{~J}$ with each pulse. Operation of the laser conformed to the hospital safety rules for laser photocoagulation.

\section{STATISTICAL METHODS}

The significance of observed differences in outcome was assessed by $\chi^{2}$ analysis with Yates's correction and Fisher's exact test when appropriate.

\section{Results}

In the 20 month period 698 patients were admitted with acute nonvariceal upper gastrointestinal haemorrhage, but 41 were not considered for entry because the trial was in abeyance owing to absence of key staff or repair and modification of laser equipment. Of the remaining 657 patients, 184 were bleeding from duodenal ulcers (118), gastric ulcers (64), or single vessels (two). One hundred and thirty of the 184 patients did not fulfil the criteria for entry into the study; bleeding in all 130 stopped with conservative management.

Fifty four patients $(29 \%)$ fulfilled the entry criteria, but nine were excluded or withdrawn for the following reasons. Two patients with bleeding from duodenal ulcers had a pylorus too narrow for duodenal intubation with the endoscope; in both the bleeding stopped with conservative management. One patient was found at endoscopy to have bled from an ulcer in the gastroduodenal region but because of distortion due to scarring the endoscopist could not decide whether the ulcer was in the stomach or the duodenum and did not see a source of bleeding in the ulcer base; the patient underwent emergency surgery because of further haemorrhage, and a duodenal ulcer of the posterior wall was found with an artery in the base. One patient fulfilled the clinical criteria but was not considered for the study because of advanced cardiac and cerebrovascular disease; he died from blood loss after endoscopy and treatment were withheld, and postmortem examination disclosed a duodenal ulcer with an artery in the base. Two patients had duodenal ulcers of the posterior wall with firm clots in the base that were not disturbed by the endoscopist. Both patients had episodes of further haemorrhage; one was not referred back for inclusion in the study, underwent emergency surgery, and survived, while the other was referred for consideration for inclusion but died from massive blood loss. The remaining three patients fulfilled the clinical criteria for entry on admission but were not referred for endoscopy until further haemorrhage had occurred. In each case brisk arterial haemorrhage was encountered at endoscopy and the procedure was abandoned without the source of bleeding being determined. Two of the patients underwent emergency surgery, after which one died; the remaining patient died owing to blood loss.

Table I gives details of the 45 patients who entered the study; 28 were men and 17 women. Their ages ranged from 22 to 88 years, with a mean of $57 \cdot 2 \pm$ SD 16.4 years; $19(42 \%)$ were over 60 . Twenty one $(47 \%)$ were shocked on admission, $31(69 \%)$ had a haemoglobin concentration on admission of less than $10 \mathrm{~g} / \mathrm{dl}$, and $44(98 \%)$ had received a blood transfusion before being included in the study. Twenty three patients were bleeding from duodenal ulcers, 20 from gastric ulcers, and two from single vessels. Arteries were considered to be the source of bleeding in five of the duodenal ulcers, 13 of the gastric ulcers, and both of the single vessels. Red, blue, or black spots were seen in 18 of the duodenal ulcers and seven of the gastric ulcers. All further analyses were made on the basis of whether arteries or spots were deemed to be the source of bleeding.

TABLE I-Details of patients entered into study

\begin{tabular}{|c|c|c|c|c|c|c|}
\hline Lesion & $\begin{array}{l}\text { Treatment } \\
\text { group }\end{array}$ & $M: F$ & $\begin{array}{c}\text { Mean ( } \pm S D) \\
\text { age (years) }\end{array}$ & $\begin{array}{l}\text { No in } \\
\text { whom } \\
\text { shock } \\
\text { present }\end{array}$ & $\begin{array}{l}\text { No in whom } \\
\text { haemoglobin } \\
<10 \mathrm{~g} / \mathrm{dl}\end{array}$ & $\begin{array}{c}\text { Mean } \\
(+ \text { SD) No } \\
\text { of units of } \\
\text { blood } \\
\text { transfused }\end{array}$ \\
\hline \multicolumn{7}{|c|}{ Bleeding from duodenal ulcer } \\
\hline $\begin{array}{l}\text { Spots } \\
\text { Arteries }\end{array}$ & $\left\{\begin{array}{l}\text { Control } \\
\text { Laser } \\
\text { Control } \\
\text { Laser }\end{array}\right.$ & $\begin{aligned} 10 & : 0 \\
8 & : 0 \\
1 & : 0 \\
2 & : 2\end{aligned}$ & $\begin{array}{c}48+21 \\
48 \pm 11 \\
60 \\
54 \cdot 5 \pm 9 \cdot 8\end{array}$ & $\begin{array}{l}3 \\
2 \\
1 \\
3\end{array}$ & $\begin{array}{l}7 \\
6 \\
1 \\
3\end{array}$ & $\begin{array}{l}2 \cdot 7 \pm 1 \cdot 4 \\
2 \cdot 8 \pm 1 \cdot 7 \\
3 \quad 3 \pm 2 \cdot 5\end{array}$ \\
\hline \multicolumn{7}{|c|}{ Bleeding from gastric ulcer } \\
\hline $\begin{array}{l}\text { Spots } \\
\text { Arteries }\end{array}$ & $\left\{\begin{array}{l}\text { Control } \\
\text { Laser } \\
\text { Control } \\
\text { Laser }\end{array}\right.$ & $\begin{array}{l}0: 6 \\
1: 0 \\
1: 5 \\
3: 4\end{array}$ & $\left.\begin{array}{c}72 \cdot 5 \pm 12 \cdot 3 \\
69 \\
69 \cdot 8 \pm 9 \cdot 8 \\
56 \cdot 4 \pm 10 \cdot 8\end{array}\right\}$ & $\begin{array}{l}4 \\
0 \\
4 \\
4\end{array}$ & $\begin{array}{l}5 \\
1 \\
4 \\
4\end{array}$ & $\begin{array}{r}2.5 \pm 1 \cdot 8 \\
2 \\
3 \cdot 2 \pm 3 \cdot 1 \\
4 \pm 1 \cdot 6\end{array}$ \\
\hline \multicolumn{7}{|c|}{ Bleeding from single vessels } \\
\hline Arteries & $\left\{\begin{array}{l}\text { Control } \\
\text { Laser }\end{array}\right.$ & $\begin{array}{l}1: 0 \\
1: 0\end{array}$ & $\begin{array}{l}60 \\
30\end{array}$ & $\begin{array}{l}0 \\
0\end{array}$ & $\begin{array}{l}0 \\
0\end{array}$ & $\begin{array}{l}4 \\
8\end{array}$ \\
\hline
\end{tabular}

$* \mathrm{p}<0.05$.

Twenty one patients were allocated to receive laser and 24 placebo treatment. The two groups were comparable in terms of age and sex for each site and source of bleeding except in the case of patients bleeding from gastric ulcers and arteries, where those allocated to receive laser treatment were younger (table I).

Table II shows the results of treatment in relation to the source of bleeding. In all 25 patients bleeding from gastric and duodenal ulcers with spots in the ulcer base the bleeding stopped with conservative management regardless of whether they were allocated to placebo or laser treatment.

TABLE II-Effect of laser photocoagulation on outcome

\begin{tabular}{|c|c|c|c|c|}
\hline & $\mathrm{n}$ & No rebleeding & $\begin{array}{l}\text { No needing } \\
\text { emergency } \\
\text { surgery }\end{array}$ & $\begin{array}{l}\text { No of } \\
\text { deaths }\end{array}$ \\
\hline \multicolumn{5}{|c|}{ Patients with spots in ulcer base } \\
\hline $\begin{array}{l}\text { Control group } \\
\text { Laser group }\end{array}$ & $\begin{array}{r}16 \\
9\end{array}$ & $\begin{array}{l}0 \\
0\end{array}$ & $\begin{array}{l}0 \\
0\end{array}$ & $\begin{array}{l}0 \\
0\end{array}$ \\
\hline Total & 25 & 0 & 0 & 0 \\
\hline \multicolumn{5}{|c|}{ Patients bleeding from arteries } \\
\hline $\begin{array}{l}\text { Control group } \\
\text { Laser: }\end{array}$ & 8 & 8 & 8 & 2 \\
\hline $\begin{array}{l}\text { Laser: } \\
\text { Used } \\
\text { Not used }\end{array}$ & $\begin{array}{l}8 \\
4\end{array}$ & $\begin{array}{l}2 \\
4\end{array}$ & $\begin{array}{l}1 \\
4\end{array}$ & $\begin{array}{l}0 \\
1 \\
\end{array}$ \\
\hline Total & 20 & 14 & 13 & 3 \\
\hline
\end{tabular}

All eight patients bleeding from arteries and allocated to receive placebo treatment experienced rebleeding and underwent emergency surgery; two died, one on the operating table owing to blood loss and the other from respiratory failure. Twelve patients with bleeding from arteries were allocated to receive laser treatment, but four did not receive it for the following reasons. In one patient with duodenal ulceration the bleeding was too brisk to permit adequate visualisation of the vessel. A second patient bleeding from a gastric ulcer would not tolerate further endoscopy to permit laser treatment after entry to the study. The remaining two patients had gastric ulcers at sites that did not allow the laser to be aimed at the arteries. All four patients had rebleeding and underwent emergency surgery; one died of sepsis and multiple organ failure. 
Of the eight patients bleeding from arteries who received laser treatment, two had rebleeding and one required emergency surgery seven days later for further bleeding. None of these eight died.

The incidence of rebleeding in patients with arterial bleeding was significantly less in those who received laser treatment than in the controls $(p<0.01)$. The incidence of emergency operations in the eight patients who received laser treatment was significantly lower than that in the controls $(\mathrm{p}<0.01)$, as was the incidence in the 12 patients allocated to receive laser treatment $(p<0.05)$. No complications occurred attributable to laser treatment. There was no significant difference between the two groups in terms of mortality or blood transfusion requirements.

\section{Discussion}

The fact that bleeding from peptic ulcers stops with conservative management means that the power of any trial of therapeutic endoscopy is weakened unless entry is restricted to those at risk of further haemorrhage. In general, patients with ulcers most likely to rebleed are those admitted with a severe index bleeding episode, ${ }^{7}$ and we were anxious to define clinical criteria for entry to identify this high risk group. Although endoscopic stigmata are of value in this regard, ${ }^{8}$ relying solely on endoscopic findings may increase the number of patients included who are at low risk of further haemorrhage. Interestingly, two previous studies that used endoscopic stigmata of recent haemorrhage as the main method of selection for trials of argon laser photocoagulation included much higher proportions of patients admitted with bleeding from peptic ulcers. $^{910}$ For example, Swain et $a l^{8}$ included 76 out of $155(49 \%)$ patients with ulcer bleeding, while the combination of clinical and endoscopic criteria used in the present study meant that only 52 out of $182(29 \%)$ patients fulfilled the entry requirements. While none of the patients of Swain et al without stigmata of recent haemorrhage had rebleeding, neither, reassuringly, did any of the 130 patients who failed to fulfil entry requirements for the present study.

Although the endoscopic stigmata of recent haemorrhage defined by Foster $e t a l^{8}$ included the presence of a vessel, the nature of the vessel concerned was not defined. In the present trial we attempted to distinguish between ulcers with arteries in the base and those with red, blue, or black spots. We recognise that such distinction is not always easy and is subject to individual interpretation, but the presence of an artery in the ulcer base was confirmed in all patients deemed to be bleeding from arteries who proceeded to surgery or died. The anatomical nature of red, blue, or black spots is controversial, but the excellent clinical progress of patients with these lesions in the present study (table II) suggests that if vessels are responsible for these endoscopic appearances they are likely to be of small calibre.

A single vessel pouting through the mucosa without obvious surrounding ulceration has been described by Jones ${ }^{11}$ and Goldman ${ }^{12}$ and was encountered in two of our patients. Although rare, this lesion accounted for $10 \%$ of the patients judged to be bleeding from arteries in the present study.

Our study showed that bleeding from gastric or duodenal ulcers with spots in the ulcer base stopped without emergency surgery regardless of the age of the patient, the severity of the index bleed, or the use of laser treatment. The laser and control groups did not differ significantly in terms of transfusion requirements. Further experience may confirm that in our practice these patients are at minimal risk of further bleeding and do not require therapeutic endoscopy or surgery. Caution must be exercised, however, as our interpretation of "spots" is similar to the definition of "vessels" used by Swain et al, ${ }^{9}$ and their experience did not appear to endorse ours.

Patients deemed to be bleeding from arteries in the present study were at major risk of further haemorrhage. All eight control patients had rebleeding and required surgery regardless of whether the artery was spurting at endoscopy, the location of the ulcer, or the patient's age. Similarly all four patients allocated to receive laser treatment but who did not receive it experienced further bleeding and required emergency operation. On the other hand, of the eight patients who received laser treatment, only two had further haemorrhage and one required urgent surgery. These findings must be interpreted in the light of our policy that the clinician responsible for patient management, while aware of the source of bleeding, remained unaware of the allocation to laser or placebo treatment.

It is difficult to compare the results of this study with others in which a neodymium yttrium aluminium garnet laser has been used. Ihre $e t a^{13}$ showed no benefit in a group of patients who had massive upper gastrointestinal haemorrhage, but no description of the sources of bleeding was given and the results in patients with bleeding from peptic ulcers cannot be interpreted. Vantrappen et $a^{14}$ showed that the neodymium yttrium aluminium garnet laser reduced the occurrence of further haemorrhage in patients with peptic ulcers with either active bleeding or fresh stigmata but they gave insufficient information on the endoscopic appearances.

The recent report from Rutgeerts et al ${ }^{15}$ showed that the neodymium yttrium aluminium garnet laser was significantly better than conservative treatment in stopping bleeding from lesions that were not spurting blood, but the reduction in the incidence of rebleeding and need for surgery in laser-treated patients was not significant. In patients with stigmata of recent haemorrhage laser treatment reduced the incidence of rebleeding and the need for surgery, but, again, the difference between the laser and control groups failed to reach statistical significance. The experience of this group in patients with active arterial spurting is difficult to evaluate since this part of their study was uncontrolled and comparison with historical controls is of limited value.

Two controlled trials of argon laser photocoagulation have been performed. ${ }^{910}$ The study conducted in Barcelona ${ }^{10}$ failed to show any benefit of treatment. Analysis of the data from the study of Swain $e t a^{9}$ shows that laser photocoagulation did not reduce the incidence of rebleeding in patients bleeding from vessels but that mortality was higher in the control group who were bleeding from vessels; this is difficult to explain.

Although the present study showed that the neodymium yttrium aluminium garnet laser reduced rebleeding and the need for emergency surgery in those bleeding from arteries, the results have to be placed in context. The arteries treated with the laser were all considered to be medium sized and did not include gastroduodenal or splenic arteries. The one patient thought to be bleeding from the gastroduodenal artery in the group allocated to laser treatment had massive active bleeding at endoscopy and was withdrawn because of an unacceptable risk of exsanguination. Because of the apparent certainty of rebleeding in patients who have bled from arteries this high risk group should be managed in surgical wards, and there is a strong case for submitting them to surgery before further haemorrhage becomes manifest if laser treatment is unavailable or inapplicable.

Although our results offer support for the contention that laser treatment is a safe and effective method of reducing the incidence of further bleeding and emergency surgery, we counsel caution in interpreting them. The technical difficulties of therapeutic endoscopy should not be underestimated. In the present study five patients were excluded or withdrawn on the grounds that the duodenum could not be entered or brisk bleeding prevented definition of the source of haemorrhage. Furthermore, three patients entered into the study with defined arterial haemorrhage did not receive their allocated laser treatment because of difficulties experienced in aiming the laser beam at the vessel responsible. Further studies in our own and other centres are needed to define the role of laser treatment with certainty and to demonstrate conclusively that mortality from ulcer haemorrhage is reduced. It must not be forgotten that therapeutic endoscopy represents, at best, a short term measure for avoiding hazardous emergency surgery and does not constitute definitive treatment for the underlying peptic ulcer disease.

We are grateful to the clinicians in Glasgow Royal Infirmary who 
allowed us to study patients under their care, and to Mrs M C Baird and Mrs $M$ Heaney for secretarial help. IAMacL is supported by a grant from Barr and Stroud Ltd.

\section{References}

${ }^{1}$ Allan R, Dykes P. A study of the factors influencing mortality rates from gastrointestinal haemorrhage. Qf Med 1976;45:533-50.

2 Jones FA. Haematemesis and melaena. Gastroenterology 1956;30:166-90.

3 Cocks JR, Desmond AM, Swynnerton BF, Tanner NC. Partial gastrectomy for haemorrhage. Gut 1972;13:331-40.

${ }^{4}$ Johnston SJ, Jones PF, Kyle J, Needham CD. Epidemiology and course of gastrointestinal haemorrhage in north-east Scotland. Br Med $\mathcal{F}$ 1973; iii: $655-60$.

5 Dronfield MW. Medical or surgical treatment for haematemesis and melaena. F R Coll Phys Lond 1979;13:84-6.

${ }^{6}$ Kiefhaber P, Nath G, Moritz K. Endoscopic control of massive gastrointestinal haemorrhage by irradiation with a high power Nd-YAG laser. Prog Surg 1977;15:140-55.

${ }^{7}$ Northfield TC. Factors predisposing to recurrent haemorrhage after acute gastrointestinal bleeding. $\mathrm{Br}$ Med $\mathcal{f} 1971 ; \mathrm{i}: 26-8$.
"Foster DN, Miloszewski KJA, Losowsky MS. Stigmata of recent haemorrhage in diagnosis and prognosis of upper gastrointestinal bleeding. Br Med f 1978;i:1173-7.

${ }^{9}$ Swain CP, Bown SG, Storey DW, Kirkham JS, Northfield TC, Salmon PR. Controlled trial of argon laser photocoagulation in bleeding peptic ulcers. Lancet 1981 ;ii:1313-6.

10 Vallon AG, Cotton PB, Laurence BM, Miro JRA, Oses SCS. Randomised trial of endoscopic argon laser photocoagulation in bleeding peptic ulcers. Gut $1981 ; 22: 228-33$.

${ }^{11}$ Jones FA. Problems of alimentary bleeding. Br Med f 1969;i:267-73.

12 Goldman RL. Submucosal arterial malformation ("aneurysm") of the stomach with fatal haemorrhage. Gastroenterology $1964 ; 46$ :589-94.

${ }^{13}$ Ihre T, Johansson C, Seligson U, Torngren S. Endoscopic YAG-laser treatment in massive upper gastrointestinal bleeding. Scand $\mathcal{F}$ Gastroenterol $1981 ; \mathbf{1 6}: 633-40$.

14 Vantrappen G, Rutgeerts P, Broeckaert L, Janssens J, Coremans G. Controlled trial of Nd-YAG laser treatment for upper digestive haemorrhage. Gastroenterology $1981 ; 80: \mathrm{A}-57$.

15 Rutgeerts P, Van Trappen G, Broeckaert L, et al. Controlled trial of YAG laser treatment of upper digestive haemorrhage. Gastroenterology 1982; $83: 410-6$.

(Accepted 1 December 1982)

\section{SHORT REPORTS}

\section{Safer insertion of pleural drains}

In recent years prepacked, sterile plastic tubes with their own central metal trocar-for example, Argyle-have become available for the insertion of pleural drains. They are said to be easy to insert ${ }^{1}$ and are claimed to be the most commonly used chest drains. ${ }^{2}$ One danger in use is the risk of sudden penetration of the trocar into the chest with consequent damage to vital structures. Such accidents are unlikely to be reported in the medical press, but probably most intrathoracic and upper abdominal organs have been penetrated at some time. ${ }^{3}$

We therefore surveyed current practice regarding chest drain insertion in the Wessex and South Western regions and have devised a simple guard for use during the procedure.

\section{Methods and results}

Current practice-All physicians with a special interest in chest disease, and thoracic surgeons in the Wessex and South Western Regional Health Authorities were asked by letter what size and type of chest drain they used to drain pneumothoraces and whether they were satisfied with the drains in use. Nineteen physicians and six surgeons replied (response rate 92\%). Eighteen used prepacked Argyle drains, and 14 of these had reservations about their use. Most were concerned about the risk of overpenetration, and several thought that they were difficult to hold and manipulate. All thoracic surgeons believed that the Argyle-type assembly was potentially dangerous, and four favoured the Tudor-Edwards trocar and cannula.

Development of guard - The purpose of the trocar is to aid passage of the drain through the chest wall. It is withdrawn once the chest wall is traversed, and the drain can be advanced with little fear of damage to vital structures. If undue force is used, however, the trocar may overpenetrate and plunge deeply within the chest. The guard was designed to prevent this overpenetration. As a preliminary, we measured the chest wall thickness in 57 cadavers (42 men and 15 women, mean ages $62(17-85)$ and $71(42-83)$ years, respectively) at the two common sites of insertion-namely, the second intercostal space in the midclavicular line, and the fourth intercostal space in the midaxillary line. Mean thicknesses at the two sites were $30 \cdot 2+$ SD 15.9 and $31.9 \pm 14.7 \mathrm{~mm}$, respectively, in the men and $29.9 \pm 10.9$ and $31.4 \pm 11.9$ $\mathrm{mm}$, respectively, in the women.

Description-The guard consists of a stainless-steel tube of $1.4 \mathrm{~cm}$ internal diameter, with a flange of $7.7 \mathrm{~cm}$ at its distal end. There are two lengths, $19 \mathrm{~cm}$ and $35 \mathrm{~cm}$, for use with $25 \mathrm{~cm}$ and $40 \mathrm{~cm}$ drains, respectively. With the drain assembly passed down the centre of the guard, the knob on the end of the trocar abuts the end of the tubular part of the guard with roughly $7 \mathrm{~cm}$ protruding distally. This distance may be varied by first inserting any, or all, of a series of nylon and molybdenum washers over the drain (figure).

Method of insertion-The skin site is prepared in the usual fashion, and using local anaesthesia a stab incision is made down to the pleura and opened. The drain and trocar assembly is passed down the centre of the guard and held with the butt end within the palm of the right hand. The palm of the left hand sits on the distal end of the guard, with the middle and index fingers straddling the protruding drain and trocar just beyond the flange (figure). Held thus, manipulation is comfortable and easy. On entering

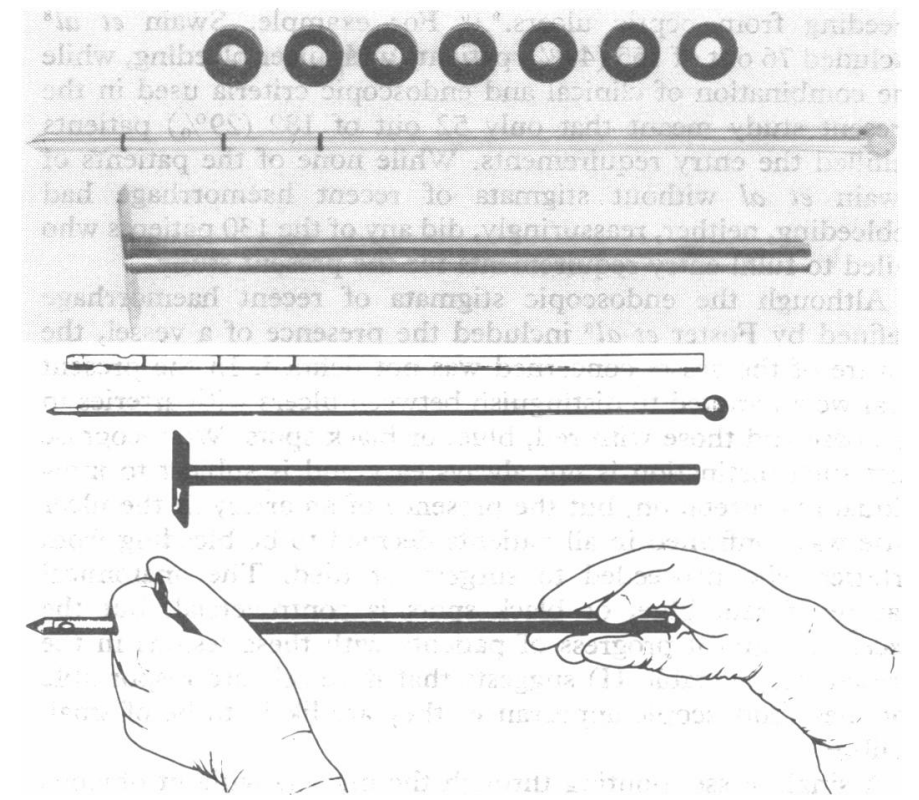

Drain and guard assembly, and method of holding assembly at insertion (no washers used).

the pleural space, the guard and trocar may be simultaneously withdrawn and the drain advanced. We have used this guard on 14 occasions with no difficulties. It has also been used by several other junior staff, including house officers. All have found it easy to use and are happier that the risk of accidental internal damage is diminished.

\section{Comment}

Thoracic surgeons in particular have asserted that safe insertion of intrapleural drains requires a formal dissection through the chest wall and pleura so that no force is used when the assembly is passed through. This view is borne out by the comments of the surgeons replying to our survey. It remains, however, a counsel of perfection and in our experience is not followed by many doctors. Chest drains are often inserted by relatively junior staff, sometimes in difficult circumstances, when the temptation to push harder than is prudent may be difficult to resist. One common method of guarding against overpenetration of the trocar is to cross-clamp the assembly with arterial forceps. ${ }^{2}$ These instruments are not designed for such purposes

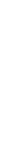

